\title{
Modification of the Oxidized Surface of Titanium to Impart of Bioactivity
}

\author{
Tatyana S Petrovskaya*and Nikita E Toropkov \\ Nanomaterials and Ceramic Engineering Group, National Research Tomsk Polytechnic University, Russia
}

*Corresponding author: Tatyana S Petrovskaya, Professor of N.M. Kizhner Research Centre, National Research Tomsk Polytechnic University, Tomsk 634050, Russia.
Received Date: June 12, 2020

Published Date: June 22, 2020

\begin{abstract}
Clinical practice has shown that the effectiveness of titanium implants increases significantly when biocompatible bioactive coatings are applied to their surface, which provide a strong bond between the implant and native bone by forming a biologically active layer of hydroxyapatite on the implant surface.

In this study ceramic-like polyoxide coatings on titanium were modified with a nanostructured sol-gel film, containing calcium, silicate and phosphate ions, which imparted hydrophilicity, solubility, roughness, adsorption activity to the surface, and increased the content of calcium and phosphate ions on the surface.

Due to new properties the surface showed bioactivity in vitro experiment. The experimental samples with a two-layer coating (polyoxide layer and sol-gel film) were kept in cell-free simulated body fluid (SBF) for 4 weeks. During experiment, the $\mathrm{pH}$ of the solution changed within the alkaline reaction, which contributed to the crystallization of calcium phosphates. On the surface of the samples, the content of calcium and phosphorus ions increased, and hydroxyapatite crystals formed.
\end{abstract}

Keywords: Titanium implants; Surface modification; Sol-gel film; Bioactivity

\section{Introduction}

The modern concept of the use of biomaterials in medicine is based on a regenerative approach, in which the emphasis is on replacing the biomaterial with native growing bone. At the same time, the restoration of the functions of the damaged bone, which carries large mechanical loads, is carried out using metal implants, among which priority is given to titanium and its alloys. The modulus of elasticity of titanium matches the modulus of bone better than the modulus of other metallic materials, which significantly reduces the implant's load on the bone. For this reason and for others, which will be discussed further, in clinical practice of orthopedics and prosthetic dentistry, titanium implants predominate.
It is well known that artificial material for the treatment and restoration of bones must meet the requirements of biocompatibility and comply with the structural-chemical and biomechanical characteristics of natural bone. Devices and prostheses made of orthopedic biomaterials should ideally work without failures throughout the patient's life. Clinical practice has shown that the effectiveness of titanium implants increases significantly when applying biocompatible bioactive coatings, which are able to ensure the creation of a single functional biomechanical implantbone system. The dynamics of osteogenesis when using titanium implants with bioactive coating is far ahead of the dynamics when using titanium covered with a bioinert coating. The terms of rehabilitation of patients may vary by 2 times. 
In contrast to bio-inert coatings based on titanium dioxide, which provide mechanical adhesion of the implant to bone, bioactive coatings containing calcium phosphates provide bioactive fixation. However, it should be noted that the practice of implantation in orthopedics and traumatology requires a differentiated approach, taking into account the type of bone tissue and the nature of the injury, age and other characteristics of the patient, the purpose and timing of the implant. Thus, the task of purposefully regulating the physicochemical properties of the coating to perform specific functions remains relevant.

Bioactive fixation is defined as the interfacial connection of the implant with the tissue through the formation of a biologically active hydroxyapatite layer on the surface of the implant.

An important advantage of bioactive fixation is that a bioactive bond is formed at the implant-bone border with a force equal to or greater than bone strength [1].

The property of bioactivity as the ability of artificial material to take part in biological processes with the formation of new bone tissue on its surface and subsequently integrate with biological bone is most often associated with calcium phosphates. Nowadays, there is agreement among researchers that bone formation is influenced by the structural, surface, physicochemical properties of calcium phosphates. In addition, the calcium and phosphate ions released by them play a significant role. They activate and stimulate natural physiological processes aimed at bone regeneration [2]. From this it follows that biomaterials, including coatings on metal, providing a gradual release of calcium and phosphate ions from the implant into the body environment and having an appropriate set of structural and surface properties, will contribute to the formation of bone tissue at the implant-biological bone interface.

\section{Discussion}

Among the methods of forming coatings on titanium, the method of micro plasma oxidation is widely used. The method provides high adhesion of the coating to titanium, which is the basis for the reliability of the medical device in operation. It allows to process implants of any shape and form ceramic-like structures in the surface layers of the metal without changing the geometric parameters of the parts. All of those are important in the manufacture of implants. The use of hydroxyapatite (HAP) in the electrolyte made it possible to obtain coatings containing calcium and phosphorus oxides in an amount of up to 13 and more than 30 wt.\% respectively [3]. Summarizing the above, it becomes obvious that an increase in the functionality of oxide coatings on titanium can be achieved by increasing the content of calcium ions in them, as well as modifying their surface in order to increase roughness, porosity, surface energy, and solubility.

In this study oxide bioactive glass synthesized by the sol-gel method, which form apatite, is considered as analogues. There was shown [1] the formation of bone-like apatite occurs on the surface of silicon dioxide obtained by the sol-gel method. A feature of these materials is the density of $\mathrm{Si}-\mathrm{OH}$ silanol groups; their presence in gel products accelerates the crystallization of hydroxyapatite, which causes an increase in the bioactivity of the material [4]. Similarly, the structural and surface properties of oxide coatings containing $\mathrm{Ca} 2+$ and P043- ions can be improved by applying sol-gel films. For this purpose, structured sol-gel films in the CaO-P205-SiO2 system were obtained and their phase composition, morphology, solubility, and behavior in SBF were studied.

Thin films up to $80 \mathrm{~nm}$ thick were applied by drawing from a colloidal solution on the polyoxide layer which has been formed on the titanium surface by micro plasma oxidation. After drying and then heat treatment at $600{ }^{\circ} \mathrm{C}$ the films are firmly bonded to the polyoxide layer of titanium surface, contain amorphous and some number of crystalline phases of $\mathrm{SiO} 2$, calcium pyrophosphate Ca2P207, and a small amount of chlorapatite. The specific surface of the outer porous layer is $18-20 \mathrm{~m} 2 / \mathrm{g}$. A nanostructured solgel film changes the morphology of the oxide layer on titanium, complementing and developing its relief. Apart from porosity the surface has roughness with an obvious two-level relief, where the parameters Ra and Rz differ by an order of magnitude.

One of the universal physicochemical characteristics of the surface of a solid is the acid-base parameter, which depends on the nature of the substance, its obtaining method and chemical composition. A study of the physicochemical properties of the surface showed that depending on the treatment conditions active centers are formed with excess positive or negative charge on the film surface, which are responsible for adsorption and chemical activity of the surface.

The experimental samples with two-layer coatings (polyoxide layer and sol-gel film) were kept in cell-free simulated body fluid (SBF). In the first two days the coating dissolves that accompanied by a loss of sample mass, and then gradual deposition of compounds from the solution occurs on its surface and that results in an increase in the mass of the sample. After 2 weeks of exposure in the SBF solution, the weight of the samples increases by $2.5 \%$, after 4 weeks - by $7.4 \%$.

While being interacting with a sample the $\mathrm{pH}$ of the solution changes within the alkaline reaction that initiates to the crystallization of calcium phosphates. On the surface of the coating, needle-shaped particles are formed; they are growing when sample is soaking in SBF, while the content of calcium and phosphorus on the surface of the sample is increasing.

Over the 4 weeks of the experiment, the concentration of calcium and phosphorous on the oxidized surface of titanium increased by 3.9 and 5.3 wt.\% (by EDXA) respectively, and on the oxidized surface with a sol-gel coating by 10 and 26.4 wt.\%, respectively. X-ray diffraction analysis revealed HAP and calcium pyrophosphate as well as chlorapatite in small amounts. 


\section{Conclusion}

A nanostructured sol-gel film containing calcium, silicate and phosphate ions being applied to titanium surface, which has been covered by polyoxide coat, modifies the surface and imparts solubility, roughness, adsorption activity to the surface, and increases the content of calcium ions on the surface. The silanol groups $\mathrm{SiOH}$ available on the sol-gel film surface are actively involved in the mineralization of the interface in

SBF environment. Such a coating is hydrophilic, intensively exchanges by calcium and phosphate ions with a solution SBF; as a result of the exchange, silanol groups bind calcium ions, contributing to the formation of a layer of amorphous and crystalline calcium phosphates.

\section{Acknowledgment}

None.

\section{Conflict of Interest}

No conflict of interest.

\section{References}

1. Sepulveda P, Jones J, Hench L (2002) Bioactive materials for tissue engineering scaffolds in: Polak J, Hench L, Kemp P, Future strategies for tissue and organ replacement. Imperial College Press, London: 3-24.

2. Jeong J, Kim JH, Shim JH, Hwang NS, Heo CY (2019) Bioactive calcium phosphate materials and applications in bone regeneration. Biomater Res 23(4).

3. Petrovskaya TS, Vereschagin VI (2015) Effectiveness of the technologies of titanium implants covering. Key Eng Materials 670: 183-188.

4. Laczka M, Cholewa K, Laczka-Osyczka A (1997) Gel-derived powders of $\mathrm{CaO}-\mathrm{P}_{2} \mathrm{O}_{5}-\mathrm{SiO}_{2}$ system as a starting material to production of bioactive ceramics. J of Alloys and Compounds 248(1-2): 42-51. 